\title{
TRANSTORNO AFETIVO BIPOLAR: AVALIAÇÃO DA SATISFAÇÃO DOS PACIENTES, FAMILIARES E PROFISSIONAIS COM O TRATAMENTO
}

Aline Esteves Pacheco ${ }^{1}$

Clarice de Lourdes Enes ${ }^{1}$

Marco Túlio Resende Clementino ${ }^{1}$

Vinicius Silva Belo ${ }^{1}$

Sebastião Júnior Henrique Duarte ${ }^{2}$

Richardson Miranda Machado ${ }^{1}$

\begin{abstract}
https://orcid.org/0000-0002-2741-4719
https://orcid.org/0000-0003-2084-0560

https://orcid.org/0000-0002-1108-935X

https://orcid.org/0000-0003-0183-1175

https://orcid.org/0000-0003-3161-9669

https://orcid.org/000-0001-9895-6905
\end{abstract}

Objetivo: Avaliar as perspectivas da satisfação dos pacientes acometidos por transtorno afetivo bipolar, seus familiares e profissionais da saúde. Metodologia: Estudo transversal com 80 indivíduos de um Centro de Atenção Psicossocial III da região Centro Oeste de Minas Gerais. Foram realizadas entrevistas, usando as Escalas de Avaliação da Satisfação com os Serviços de Saúde Mental. Resultados: Os índices de satisfação apresentaram-se maiores entre pacientes, especialmente no domínio Acolhida e ajuda recebida no serviço [escore 4,66; n = 30 (83,3\%)]. Entre os familiares, o maior índice de satisfação foi no domínio Resultados do tratamento [escore 4,66; $\mathrm{n}=27(79,4 \%)$ ]. O destaque dos profissionais foi na insatisfação, referente ao domínio Condições de trabalho [escore 2,85; n = 7 (70\%)]. Conclusão: Constatou-se que os pacientes estão satisfeitos com o serviço e suas perspectivas de satisfação são as maiores, seguidos dos familiares e profissionais.

Descritores: Transtorno Afetivo Bipolar, Satisfação do Paciente, Cuidadores Familiares, Satisfação Profissional, Qualidade da Assistência à Saúde.

\section{BIPOLAR AFFECTIVE DISORDER: ASSESSMENT OF PATIENT, FAMILY AND PROFESSIONAL SATISFACTION WITH TREATMENT}

Objective: To evaluate the perspectives of satisfaction of patients affected by bipolar affective disorder, their families and health professionals. Methodology: Cross-sectional study with 80 individuals from a Psychosocial Care Center III in the Midwest region of Minas Gerais. Interviews were conducted using the Satisfaction Rating Scales with Mental Health Services. Results: Satisfaction rates were higher among patients, especially in the domain Welcoming and help received at the service [score 4.66; $n=30$ (83.3\%)]. Among family members, the highest satisfaction rate was in the treatment results domain [score 4.66; $n=27(79.4 \%)$ ]. The highlight of the professionals was dissatisfaction, referring to the domain Working conditions [score 2.85; $n=7(70 \%)$. Conclusion: It was found that patients are satisfied with the service and their satisfaction prospects are the highest, followed by family members and professionals.

Descriptors: Bipolar Affective Disorder, Patient Satisfaction, Family Caregivers, Professional Satisfaction, Quality of Health Care.

TRASTORNO AFECTIVO BIPOLAR: EVALUACIÓN DE LA SATISFACCIÓN DEL PACIENTE, LA FAMILIA Y EL PROFESIONAL CON EL TRATAMIENTO

Objetivo: evaluar las perspectivas de satisfacción de los pacientes afectados por el trastorno afectivo bipolar, sus familias y profesionales de la salud. Metodología: Estudio transversal con 80 personas de un Centro de Atención Psicosocial III en la región del Medio Oeste de Minas Gerais. Las entrevistas se realizaron utilizando las escalas de calificación de satisfacción con los servicios de salud mental. Resultados: las tasas de satisfacción fueron más altas entre los pacientes, especialmente en el dominio de bienvenida y la ayuda recibida en el servicio [puntuación 4.66; $n=30$ (83,3\%)]. Entre los miembros de la familia, la tasa de satisfacción más alta se encontraba en el dominio de resultados del tratamiento [puntuación 4.66; n = 27 (79,4\%)]. Lo más destacado de los profesionales fue la insatisfacción, refiriéndose al dominio Condiciones de trabajo [puntaje 2.85; $\mathrm{n}=7$ (70\%)] Conclusion: Se encontró que los pacientes están satisfechos con el servicio y sus perspectivas de satisfacción son las más altas, seguidas por los familiares y profesionales.

Descriptores: Trastorno Afectivo Bipolar, Satisfacción del paciente, Cuidadores familiares, Satisfacción professional, Calidad de la atención médica.

Universidade Federal de São João Del Rei, MG, Brasil.

${ }^{2}$ Universidade Federal de Mato Grosso do Sul, MT, Brasil.

Autor Correspondente: Esteves Pacheco Email:linesteves@yahoo.com.br

Recebido: 02/12/19 Aceito:18/4/20 


\section{INTRODUÇÃO}

O Transtorno Afetivo Bipolar (TAB) é grave, multifatorial, crônico, progressivo e associado a altos níveis de incapacitação e morbidade ${ }^{1}$. Atualmente, é a sexta maior causa de incapacidade em jovens, podendo desencadear problemas cognitivos, comprometimento funcional e aumento da mortalidade, particularmente por suicidio e doença cardiovascular - responsável pela redução de dez a vinte anos na expectativa de vida dos pacientes ${ }^{2}$. Por isso, a detecção precoce é fundamental, para evitar a progressão da doença, o impacto sobre a funcionalidade do indivíduo e a recorrência dos episódios agudos ${ }^{3}$. O tratamento envolve medicamentos estabilizadores do humor, acompanhamento clínico e psicossocial, aliado a estratégias terapêuticas que contemplem a multifatorialidade do transtorno, considerando a alteridade do sujeito, com a desconstrução de práticas de objetivação da doença mental, como acontece em Centros de Atenção Psicossociais (CAPS) ${ }^{4-5}$.

Por isso os CAPS foram escolhidos para desenvolvimento deste estudo, já que estes serviços vêm assumindo especial relevância nas práticas em saúde mental no país ${ }^{6.7}$. A avaliação das pessoas envolvidas neste contexto é necessária para monitorar o tratamento e corrigir eventuais aspectos negativos, a fim de aumentar a adesão, evitar novas crises (sofrimento físico e emocional) e novos custos para o paciente e para o serviço, pois, a satisfação, aliada ao apoio familiar e dos profissionais de saúde, aumenta significativamente a adesão e a efetividade das ações. Além disso, como qualquer tratamento de doença crônica, o do TAB precisa de acompanhamento próximo, oportunizando ajustes periódicos das necessidades ${ }^{8}$.

O objetivo desta pesquisa foi avaliar as perspectivas da satisfação dos pacientes acometidos por transtorno afetivo bipolar, seus familiares e profissionais da saúde, considerando que a avaliação simultânea da satisfação desta tríade constata um nível de satisfação mais apurado.

Considerando esta medida um importante critério para avaliação da qualidade do tratamento oferecido, o fato de ser realizada de forma integrada demonstra maior confiabilidade, possibilitando melhor planejamento da assistência.

\section{METODOLOGIA}

\section{Tipo de estudo}

Transversal e descritivo.

\section{Participantes da pesquisa}

A população foi composta por 80 participantes, sendo 36 pacientes, 34 familiares e 10 profissionais da saú- de, referências técnicas que atuam no serviço. Todos os 37 pacientes com diagnóstico de TAB que foram atendidos naquele local, na modalidade de tratamento permanência-dia, foram convidados e um recusou participar. A partir daí, 36 familiares foram contatados, sendo que 34 foram encontrados e aceitaram participar da pesquisa. Não houve perda entre os profissionais, pois todos os que estão no serviço responderam à entrevista.

\section{Local do estudo}

Realizado no CAPS III do município de Divinópolis, Minas Gerais, no período de 01 de fevereiro de 2017 a 01 de fevereiro de 2018.

\section{Coleta dos dados}

Foram utilizadas as três versões da "Escala de Avaliação da Satisfação com os Serviços de Saúde Mental (SATIS-BR)" - para pacientes, familiares e profissionais da saúde. Tais escalas são de acesso livre e gratuito e foram previamente validadas no Brasil, tendo seus itens agregados por domínios e separados em subescalas.

Cada subescala do instrumento tem seu escore calculado individualmente, o que permitiu avaliar o nível de satisfação em cada domínio, onde a pontuação foi obtida por meio de uma escala do tipo Likert de cinco pontos (1 a 5).

\section{Procedimentos de análise dos dados}

Os dados obtidos foram analisados no software R versão 3.5.1. A normalidade dos dados quantitativos foi analisada pelo teste de Shapiro-Wilk. Tendo em vista a distribuição não normal, foram calculados os valores das medianas de cada escala e conduzidos testes não paramétricos, para analisar a existência de diferenças globais entre os valores das medianas das subescalas, e para identificar quais pares de subescalas apresentavam diferenças estatisticamente significativas entre si. Em todas as comparações, adotou-se um nivel de significância estatística de 95\%.

\section{Procedimentos éticos}

O projeto de pesquisa foi aprovado pelo Comitê de Ética em Pesquisa da Universidade Federal de São João Del-Rei (UFSJ/CCO) sob o no 1.868.647. Conforme previsto, os pacientes foram convidados a participar da pesquisa assim que estavam prontos para alta do serviço e, somente após esclarecimentos e assinatura do Termo de Consentimento Livre e Esclarecido (TCLE), os questionários eram aplicados pelos pesquisadores. Caso o paciente aceitasse, seu familiar e o profissional responsável também eram abordados e esclarecidos em relação à pesquisa. Conforme aceitavam, assinavam o TCLE específico e eram entrevistados. 


\section{RESULTADOS}

Tabela 1 - Caracterização sócio demográfica de pacientes com transtorno afetivo bipolar, seus familiares e profissionais do CAPS III. Divinópolis, MG, Brasil, 2018.

\begin{tabular}{|c|c|c|c|}
\hline Variáveis Sociodemográficas & $\begin{array}{c}\text { Pacientes } \\
n=36(\%)\end{array}$ & $\begin{array}{c}\text { Familiares } \\
n=34(\%)\end{array}$ & $\begin{array}{c}\text { Profissionais } \\
n=10(\%)\end{array}$ \\
\hline \multicolumn{4}{|l|}{ Sexo } \\
\hline Masculino & $12(33,3)$ & $15(44,1)$ & $5(50,0)$ \\
\hline Feminino & $24(66,7)$ & $19(55,8)$ & $5(50,0)$ \\
\hline \multicolumn{4}{|l|}{ Faixa Etária } \\
\hline 20 a 29 anos & $3(8,3)$ & $4(11,8)$ & - \\
\hline 30 a 39 anos & $6(17,7)$ & $9(26,5)$ & - \\
\hline 40 a 49 anos & $12(33,3)$ & $6(17,6)$ & $5(50,0)$ \\
\hline 50 a 59 anos & $8(22,2)$ & $5(14,7)$ & $5(50,0)$ \\
\hline 60 anos e mais & $7(19,4)$ & $10(29,4)$ & - \\
\hline \multicolumn{4}{|l|}{ Estado civil } \\
\hline Solteiro & $14(38,9)$ & $5(14,7)$ & - \\
\hline Casado/Amasiado & $11(30,6)$ & $20(58,8)$ & $10(100)$ \\
\hline Separado/Divorciado & $10(27,8)$ & $5(14,7)$ & - \\
\hline Viúvo & $l(2,8)$ & $4(11,8)$ & - \\
\hline \multicolumn{4}{|l|}{ Escolaridade } \\
\hline Nenhuma & - & $2(5,9)$ & - \\
\hline Ensino Fundamental & $21(58,3)$ & $18(52,9)$ & - \\
\hline Ensino Médio & $9(25,0)$ & $7(20,6)$ & - \\
\hline Ensino Superior & $6(16,7)$ & $7(20,6)$ & $10(100)$ \\
\hline
\end{tabular}

Foi apresentado um escore de satisfação global dos sujeitos e os escores por domínios, com base na classificação adotada, considerando: muito insatisfeito e insatisfeito (1-2), mais ou menos satisfeito (3) e satisfeito e muito satisfeito (45). Este agrupamento foi feito devido ao fato de que alguns escores tinham poucos indivíduos e a categorização repre- sentou melhor o padrão de satisfação dos grupos analisados.

A média de todas as respostas que compunham cada escala e cada subescala resultou em valores dos escores finais da satisfação. Quanto maior a pontuação obtida em cada domínio, maior foi o grau de satisfação com o serviço, naquele contexto. 
Para os pacientes (Tabela 2) houve valores significativamente maiores $(p<0,001)$ para o domínio 2 [Acolhida da equipe e ajuda recebida no serviço] em relação aos de- mais, e não houve diferenças entre os domínios 1 [Competência e compreensão da equipe] e 3 [Condições físicas e conforto do serviço].

Tabela 2 - Distribuição da frequência dos pacientes com TAB (n=36) quanto à avaliação da satisfação com o tratamento recebido no CAPS III. Divinópolis, MG, Brasil, 2018.

\begin{tabular}{|c|c|c|c|c|c|c|c|c|}
\hline \multirow{3}{*}{ Satisfação por Domínios } & \multirow{2}{*}{\multicolumn{2}{|c|}{ Insatisfeito }} & \multicolumn{2}{|c|}{ Mais ou menos } & \multirow{2}{*}{\multicolumn{2}{|c|}{ Satisfeito }} & \multirow[b]{2}{*}{ Média } & \multirow[b]{2}{*}{ Medianz } \\
\hline & & & & eito & & & & \\
\hline & $n$ & $\%$ & $\mathrm{n}$ & $\%$ & $n$ & $\%$ & - & - \\
\hline $\begin{array}{l}\text { Dominio } 1 \text { (competência e com- } \\
\text { preensão da equipe) }\end{array}$ & 1 & 2,8 & 17 & 47,2 & 18 & 50,0 & 3,92 & 3,91 \\
\hline $\begin{array}{l}\text { Dominio } 2 \text { (acolhida da equipe e } \\
\text { ajuda recebida no serviço) }\end{array}$ & 2 & 5,6 & 4 & 11,1 & 30 & 83,3 & 4,47 & 4,66 \\
\hline $\begin{array}{l}\text { Dominio } 3 \text { (condições físicas e con- } \\
\text { forto do serviço) }\end{array}$ & 7 & 19,4 & 10 & 27,8 & 19 & 52,8 & 3,51 & 4,00 \\
\hline Satisfação Global & & & & & & & 4,18 & 4,2 \\
\hline
\end{tabular}

Para os familiares houve valores significativamente maiores no domínio 1 [Resultados do tratamento no serviço], em relação ao domínio 3 [Privacidade e confidencialidade do serviço] ( $p<0,001)$, e não houve diferenças nas demais comparações entre os pares.

Tabela 3 - Distribuição da frequência dos familiares dos pacientes com TAB (n=34*) quanto à avaliação da satisfação com o tratamento recebido pelo paciente no CAPS III. Divinópolis, MG, Brasil, 2018.

\begin{tabular}{|c|c|c|c|c|c|c|c|c|}
\hline \multirow{3}{*}{ Satisfação por Domínios } & \multirow{2}{*}{\multicolumn{2}{|c|}{ Insatisfeito }} & \multicolumn{2}{|c|}{ Mais ou menos } & \multirow{2}{*}{\multicolumn{2}{|c|}{ Satisfeito }} & \multirow[b]{2}{*}{ Média } & \multirow[b]{2}{*}{ Mediana } \\
\hline & & & \multicolumn{2}{|c|}{ satisfeito } & & & & \\
\hline & $n$ & $\%$ & $n$ & $\%$ & $n$ & $\%$ & - & - \\
\hline $\begin{array}{l}\text { Domínio } 1 \text { (resultados do trata- } \\
\text { mento no serviço) }\end{array}$ & 2 & 5,9 & 05 & 14,7 & 27 & 79,4 & 4,36 & 4,66 \\
\hline $\begin{array}{l}\text { Domínio } 2 \text { (acolhida e competên- } \\
\text { cia da equipe) }\end{array}$ & 4 & 11,7 & 05 & 14,7 & 25 & 73,6 & 4,04 & 4,00 \\
\hline $\begin{array}{l}\text { Dominio } 3 \text { (privacidade e confiden- } \\
\text { cialidade do serviço) }\end{array}$ & 4 & 11,7 & 04 & 11,7 & 26 & 76,6 & 3,86 & 4,00 \\
\hline Satisfação Global & & & & & & & 4,12 & 4,37 \\
\hline
\end{tabular}


Em relação aos profissionais, o domínio 3 [Condições de trabalho] apresentou os menores valores. Estes foram significativamente menores do que os do domínio 4 [Relacionamento no serviço, com colegas e superiores] $(p=0,019)$ e, nas demais comparações, não houve diferenças significativas. Os profissionais entrevistados tinham formação em serviço social, psicologia e terapia ocupacional.

Tabela 4 - Distribuição da frequência dos profissionais $(n=10)$ quanto à avaliação da satisfação com seu trabalho e com o tratamento oferecido ao paciente com TAB, no CAPS III. Divinópolis, MG, Brasil, 2018.

\begin{tabular}{|c|c|c|c|c|c|c|c|c|}
\hline \multirow[t]{2}{*}{ Satisfação por Domínios } & \multicolumn{2}{|c|}{ Insatisfeito } & \multicolumn{2}{|c|}{$\begin{array}{c}\text { Mais ou menos } \\
\text { satisfeito }\end{array}$} & \multicolumn{2}{|c|}{ Satisfeito } & \multirow{2}{*}{$\begin{array}{c}\text { Média } \\
- \\
\end{array}$} & \multirow{2}{*}{$\begin{array}{c}\text { Mediana } \\
- \\
\end{array}$} \\
\hline & $\mathbf{n}$ & $\%$ & $\mathrm{n}$ & $\%$ & $\mathbf{n}$ & $\%$ & & \\
\hline $\begin{array}{l}\text { Domínio } 1 \text { (qualidade dos serviços } \\
\text { oferecidos aos pacientes) }\end{array}$ & 1 & 10,0 & 09 & 90,0 & 0 & 0,0 & 3,43 & 3,45 \\
\hline Domínio 2 (participação no serviço) & 3 & 30,0 & 07 & 70,0 & 0 & 0,0 & 3,22 & 3,28 \\
\hline Domínio 3 (condições de trabalho) & 7 & 70,0 & 03 & 30,0 & 0 & 0,0 & 2.73 & 2,85 \\
\hline $\begin{array}{l}\text { Domínio } 4 \text { (relacionamento no ser- } \\
\text { viço, com colegas e superiores) }\end{array}$ & 3 & 30,0 & 04 & 40,0 & 03 & 30,0 & 3.46 & 3,50 \\
\hline Satisfação Global & & & & & & & 3,15 & 3,15 \\
\hline
\end{tabular}

\section{DISCUSSÃO}

Esta pesquisa confirmou a realidade dos sistemas de saúde, na qual o número de mulheres que procura atendimento é consideravelmente maior. Apesar do reduzido número de pesquisas de gêneros comprometer o atendimento de necessidades específicas, considerando a existência de fatores inerentes que influenciam os cuidados, mulheres parecem ter melhor funcionamento global em relação à gravidade da doença e ao convívio social ${ }^{8,5}$.

Em grupos de menor faixa etária o diagnóstico é difícil, pois os sintomas iniciais são inespecificos e exigem uma avaliação clínica cuidadosa. O manejo ideal requer estratégias preventivas que incluam terapias psicológicas e abordagem do estilo de vida, desde o início dos sintomas. O ideal seria prevenir o agravamento das consequências e extensão da doença, já que pode haver associação entre comportamento e estilo de vida das/ os pacientes ${ }^{2,9-10}$. Conforme a estratégia defendida pela Reforma Psiquiátrica, a comunicação com a Rede de Atenção à Saúde deve ser eficaz, possibilitando intervenções precoces na lógica do território de residênciall.

Quanto à escolaridade, certamente, pessoas com melhor desempenho escolar utilizam menos os serviços públicos para tratamento, devido à maior estabilidade do quadro ${ }^{8}$, apesar de apresentarem maior risco de desenvolverem a doença ${ }^{12}$. Já o estado civil predominante pode ser resultado das dificuldades de relacionamento geradas a partir das crises. Acredita-se haver relação causal entre estas e o rompimento de relações, 
em razão das tensões impostas aos casais que separam, quando sobrecarregados ${ }^{6}$.

Entre familiares, o maior número de pessoas com mais de 40 anos do sexo feminino, corroborou com outro estudo ${ }^{8}$, sendo a faixa etária que dispõe de mais tempo para acompanhar o familiar que está em tratamento. Talvez a essência feminina de cuidar seja aspecto relevante para assumirem este papel nas relações familiares, além de questões de aceitação e prática religiosa também surgirem como estratégias de enfrentamento utilizadas ${ }^{13}$. Entretanto, é necessária a expansão da compreensão das características de qualidade, custos e benefícios do cuidado familiar no longo prazo ${ }^{14}$. A questão precisa ser ampliada para a carência de cuidados abrangentes, pois, na maioria das vezes, os familiares não são especialistas e não têm acesso fácil a estes.

O estado civil desses familiares foi muito próximo aos dados encontrados em estudo similar ${ }^{8}$, compatível com o melhor desempenho do papel de cuidador quando o familiar usufrui de uma relação partilhada do cuidado, minimizando o impacto da sobrecarga. Porém, os familiares exigem atenção especial do serviço de saúde, pois o diagnóstico de TAB compromete os membros da família com auto sacrifício, excessiva carga de cuidado e grande impacto emocional. Precisam receber auxílio para acessarem informações úteis para o enfrentamento das alterações da rotina, conciliando cuidados prestados e o autocuidado $0^{6,14-15}$

Os profissionais entrevistados desenvolviam suas atividades de forma interdependente, em prol do projeto terapêutico único para cada paciente. Estes estavam equiparados quanto ao sexo, e não foram observadas diferenças significativas de trabalho entre gêneros, apesar de não terem sido pesquisadas situações externas, que possam diferenciar os processos de vida dos sujeitos. Sugere-se que outras pesquisas considerem os riscos psicossociais resultantes da sobrecarga da conciliação do trabalho e da vida pessoal, investigando possibilidades de intervenção, a fim de melhorar o bem-estar dessas pessoas ${ }^{16}$.

A média de tempo gasta para alocação e estabilização de profissionais no mercado de trabalho foi condizente com a faixa etária encontrada. A expertise é um fator que contribui para qualidade do tratamento, pois quanto maior a experiência, maior destreza e percepção desse profissional em relação aos componentes técnicos do processo de cuidado, em prol dos pacientes, e maior a credibilidade no trabalho que desenvolvem, minimizando possiveis entraves interpessoais advindos da doença mental 3.6

De fato, os pacientes sentiram-se satisfeitos com a acolhida e ajuda recebidas, tendo se destacado o fato de terem se sentido respeitados, ao serem ouvidos com atenção pelo profissional que os admitiu, sentindo-se compreendidos. Obtiveram o tipo de ajuda que necessitavam e consideraram a equipe competente, em consonância com outros estudos ${ }^{8,17}$. A satisfação encontrada pode indicar a consolidação dos princípios da Reforma Psiquiátrica no quesito da formação e atuação profissional, que se mostrou capaz de assegurar uma prestação digna de cuidados a partir das construções práticas da dimensão assistencial, as quais possuem grande repercussão na consolidação da autonomia profissional ${ }^{18}$

Estes pacientes apresentaram-se insatisfeitos com as condições físicas e conforto, sendo itens que tiveram menores escores de satisfação "aparência do serviço" e "condições gerais" das instalações. Os dados evidenciam uma grande fragilidade do CAPS relacionada às suas condições de infraestrutura, convergindo com um estudo realizado no sul do $\mathrm{Brasil}^{8}$ e divergindo de outro estudo realizado no estado do Rio de Janeiro, quando foram considerados satisfatórios todos os índices de avaliação com o serviço oferecido ${ }^{19}$. Mas, em geral, os serviços substitutivos ao antigo cenário manicomial têm recebido baixos investimentos financeiros, dificultando a instalação e manutenção da estrutura física adequada.

Outros autores apontaram que maiores escores de satisfação foram associados a pacientes em tratamento pelo Sistema Único de Saúde (SUS) e a pessoas com menor escolaridade ${ }^{20}$. Mas, neste estudo, a maioria dos pacientes se referiu mais ou menos satisfeita, indicando a necessidade de melhorias no serviço, para se constituir como referência e ser reconhecido como dispositivo substitutivo à lógica hospitalar.

Entre familiares, também foram evidenciados niveis de satisfação, mantendo a mesma relação média entre os itens distribuídos nos domínios. Satisfeitos com o serviço prestado consideraram que o tratamento dispensado era necessário e estava ajudando o paciente a lidar com seus problemas, corroborando com alguns estudos ${ }^{8,22}$, mas contrapondo outro, no qual havia uma grande lacuna entre as expectativas e percepções das famílias sobre o apoio real oferecido pelos profissionais ${ }^{21}$. As maiores médias de satisfação referiram-se às medidas de privacidade e confidencialidade, evidenciando que os profissionais atuam com ética, oferecendo segurança e confiança no manuseio e exposição das informações. 
No entanto, os familiares estavam insatisfeitos com a infraestrutura e com o número de atendimentos individuais dos pacientes, reforçando a necessidade de maiores investimentos estruturais e em recursos humanos, adequados ao atendimento da crescente demanda em saúde mental. Possivelmente, familiares de pacientes com TAB possuem angústias e expectativas específicas, como foi o caso da maior preocupação manifestada, referente à segurança física e o futuro dos pacientes, pois os dados contestaram um estudo desenvolvido em um CAPSIII na região sul do Brasil, com pessoas diagnosticadas com outros transtornos psiquiátricos ${ }^{8}$.

A maioria dos familiares estava mais ou menos satisfeita no escore global, diferentemente de outro estudo com o mesmo instrumento, desenvolvido em um CAPSi do interior de Minas Gerais ${ }^{23}$. Decerto, ainda apresentam dificuldades em compreender a lógica de tratamento proposta pela Reforma Psiquiátrica, que reinsere família e sociedade no processo de tratamento. O fato de o familiar ser inquerido a participar do tratamento e da avaliação da melhora apresentada pelo paciente, com intervenções específicas, pode gerar baixa satisfação.

No escore da satisfação global, a maioria dos profissionais referiu-se mais ou menos satisfeita com o serviço. Apresentaram-se insatisfeitos com a qualidade dos serviços oferecidos, participação da equipe, expectativas de serem promovidos, salário e condições de trabalho, reiterando outra pesquisa que identificou escore de satisfação média entre profissionais de um CAPS na cidade do Rio de Janeiro ${ }^{16}$. Alegaram satisfação somente nos relacionamentos interpessoais com colegas e na autonomia no trabalho.

Os dados obtidos nesse estudo contradisseram os de outros estudos brasileiros em relação ao trabalho e competência da equipe e aos cuidados dispensados aos pacientes. Apesar disso, todos os autores destacam que os serviços precisam de maiores investimentos, qualificação profissional e melhorias organizacionais, como reavaliação das carreiras, pois a satisfação profissional é um indicador da qualidade do serviço e o cuidado oferecido é reflexo desses ajustes ${ }^{16,22-24}$.

A maioria dos pacientes, familiares e profissionais apresentaram insatisfação com a estrutura do serviço, mas a compreensão da equipe sobre as necessidades do paciente e a qualidade dos cuidados dispensados aos mesmos foram dois aspectos que geraram satisfação. Pacientes e profissionais concordaram que o tipo de tratamento oferecido, de informação dada e a competência da equipe são satisfatórias, em relação à qual, familiares acataram. Apesar do pouco conhecimento que as pessoas têm dos seus direitos sociais, não foi constatada a presença de aceitabilidade social, pois altos valores de satisfação não foram encontrados, e, por isso, o risco de viés de gratidão foi excluído daquele cenário 22,24-25.

\section{Limitações do estudo}

Observamos dificuldades em relação aos questionários utilizados devido ao grande número de perguntas e uso de termos que dificultavam a compreensão dos entrevistados. Apesar disso, os instrumentos utilizados cumpriram seu papel e foram de fácil aplicabilidade na forma presencial.

\section{Contribuições para a Prática}

O estudo contribui com conhecimentos que podem melhorar o diálogo entre os sujeitos que usam e atuam no serviço de atenção psicossocial. À medida que forem criadas alternativas para amenizar as características negativas mais relevantes, os principais aspectos de insatisfação apontados pelos participantes poderão ser discutidos, impactando positivamente no serviço. Os profissionais também poderão planejar melhor o tratamento e, a partir da estratégia de ampliação das vozes dos familiares e pacientes, aumentar a satisfação destes.

\section{CONCLUSÃO}

A aplicação simultânea dos três questionários, de forma inédita neste tipo de serviço, garantiu a presente perspectiva de avaliação e apresentou-se como nova possibilidade de reflexão sobre o processo da Reforma Psiquiátrica no Brasil, implantação e efetividade dos serviços abertos, importantes para o avanço do cuidado em saúde mental.

Sugere-se que este cenário seja explorado quanto às expectativas envolvidas nas relações entre os serviços e usuários, e entre profissionais e serviços, para melhor entendimento da forma e medida em que a Política de Saúde Mental está sendo implementada.

Apesar de a avaliação da satisfação com o serviço envolvendo as três dimensões ter possibilitado uma compreensão mais abrangente e integrada da realidade observada, esta pesquisa abre novas possibilidades de perguntas, visando agregar e aprofundar o conhecimento a esse respeito. Como não foram encontrados resultados de muita satisfação, os pontos negativos levantados pelos participantes precisam ser melhor estudados.

Contribuições dos Autores: Todos os autores participaram das etapas do estudo. 


\section{REFERÊNCIAS}

1. Panizzutti BS. O papel dos marcadores biológicos como indicativos de envelhecimento precoce no transtorno bipolar. [Internet]. Porto Alegre: Universidade Federal do Rio Grande do Sul; 2016 [cited 2019 Set 30]. Available from: http://www.lume.ufrgs.br/handle/10183/197249 2. Vieta E, Berk M, Schulze TG, Carvalho AF, Suppes T, Calabrese JR, et al. Bipolar disorders. Nature Reviews Disease Primers [Internet]. 2018 [cited 2019 Out 02];4(18008): [about lp.]. Available from: https://www.ncbi.nlm.nih.gov/pubmed/29516993

3. Mccormick U, Murray B, Mcnew B. Diagnosis and treatment of patients with bipolar disorder: A review for advanced practice nurses. J Am Assoc Nurse Pract. 2015 [cited 2018 Jan 18];27(9):530-42. Available from: https://www.ncbi.nlm.nih.gov/pmc/articles/PMC5034840/

4. Fountoulakis KN, Vieta E, Young A, Yatham L, Grunze H, Blier P, et al. The International College of Neuropsychopharmacology (CINP) Treatment Guidelines for Bipolar Disorder in Adults (CINP-BD-2017), Part 4: Unmet Needs in the Treatment of Bipolar Disorder and Recommendations for Future Research. Int J Neuropsychopharmacol [Internet]. 2017 [cited 2018 Nov 24];20(2):196-205. Available from: https://academic. oup.com/ijnp/article/20/2/196/2629254/

5. Tavares CM. Mesquita LM. Sistematização da assistência de enfermagem e clínica ampliada: desafios para o ensino de saúde mental. Enferm Foco [Internet]. 2019; 10(7): 121-126. Available from: http://revista.cofen.gov.br/index.php/enfermagem/article/view/2810/560

6. Granek L, Danan D. Bersudsky Y, Osher Y. Living with bipolar disorder: the impact on patients, spouses, and their marital relationship. Bipolar Disord [Internet]. 2016 [cited 2018 Mar 30];18(2):192-9. Available from: http://www.ncbi.nlm.nih.gov/pubmed/26890335

7. Miziou S, Tsitsipa E, Moysidou S, Karavelas V. Dimelis D. Polyzoidou V, et al. Psychosocial treatment and interventions for bipolar disorder: a systematic review. Ann Gen Psychiatry. [Internet]. 2015 [cited 2018 Nov 24];14(19):1-11. Available from: https://www.ncbi.nlm.nih.gov/pmc/ articles/PMC4493813/

8. Resende KIDS de, Bandeira M, Oliveira DCR. Assessment of Patient, Family and Staff Satisfaction in a Mental Health Service. Paidéia [Internet]. 2016 Aug [cited 2018 May 29]:26(64):245-53. Disponivel em: http://www.scielo.br/scielo.php?script=sci_arttextEpi$\mathrm{d}=\mathrm{S} 0103-863 \times 2016000200245 \varepsilon \operatorname{lng}=\mathrm{en} \varepsilon t \operatorname{lng}=\mathrm{en}$

9. Magalhães PV da S, Costa MH, Pinheiro RT. Epidemiologia do transtorno bipolar. In: Transtorno Bipolar: teoria e clinica. Porto Alegre: Artmed Editora Ltda.; 2015. p. 21-31.

10. Solé B, Jiménez E, Torrent C, Reinares M, Bonnin CdelM. Torres I, et al. Cognitive Impairment in Bipolar Disorder: Treatment and Prevention Strategies. Int J Neuropsychopharmacol [Internet]. 2017 [cited 2019 Set 30]; 20(8):670-680. Available from: http:// doi: 10.1093/ijnp/ pyx032

11. Galvanese ATC, D'Oliveira AFPL, Lima EMFA, Pereira LMF, Nascimento AP, Nascimento AF. Arte, saúde mental e atenção pública: traços de uma cultura de cuidado na história da cidade de São Paulo. Hist. cienc. Saude-Manguinhos [Internet]. 2016 [acesso em: 06 out. 2019]: 23(2):431-452 Available from: http://www.scielo.br/scielo.php?pid=S0104-59702016000200431Escript=sci_arttext

12. Peters AT, E. WA, Eisner L, Baek JH, Deckersbach T. The burden of repeated mood episodes in bipolar I disorder: Results from the National Epidemiological Survey on Alcohol and Related Conditions (NESARC). J Nerv Ment Dis. [Internet]. 2016 [cited 2018 Jun 10]:204(2):87-94. Available from: https://www.ncbi.nlm.nih.gov/pmc/articles/PMC4733595/

13. Iseselo MK, Kajula L, Yahya-Malima KI. The psychosocial problems of families caring for relatives with mental illnesses and their coping strategies: a qualitative urban based study in Dar es Salaam, Tanzania. BMC Psychiatry [Internet]. 2016:16(146):1-12. doi.org/10.1186/s12888016-0857-y

14. Malec JF, Van Houtven CH, Tanielian T, Atizado A, Dorn MC. Impact of TBI on caregivers of veterans with TBI: Burden and interventions. Brain Inj [Internet]. 2017;31(9):1235-45. doi.org/10.1080/02699052.2016.1274778

15. Pocinho R, Belo P. Melo C, Navarro-Pardo E, Muñoz JJF. Relação entre o estado psicossocial do cuidador informal e o tempo de cuidado dos idosos da região centro de Portugal. Rev Educ y Humanismo [Internet]. 2017; 19(32):88-101. doi.org/10.17081/eduhum.19.32.2533

16. Ferreira AP. Satisfação, sobrecarga de trabalho e estresse nos profissionais de serviço de saúde mental. Rev Bras Med Trab [Internet] 2015 [cited 2019 Set 30]; 13(2). Available from: https://www.arca.fiocruz.br/handle/icict/15168

17. Silva SN, Lima MG, Ruas CM. Avaliação de Serviços de Saúde Mental Brasileiros: satisfação dos usuários e fatores associados. Cien Saude Colet [Internet]. 2018:23(11):3799-810. Available from: https://www.scielosp.org/pdf/csc/2018.v23n1l/3799-3810/pt

18. Pinheiro CW, Araújo MAM, Rolim KMC, Oliveira CM, Alencar AB. Teoria das relações interpessoais: reflexões acerca da função terapêutica do enfermeiro em saúde mental. Enferm Foco [Internet]. 2019; 10(3):64-69. Available from: http://revista.cofen.gov.br/index.php/enfermagem/article/view/2291/580

19. Barcelos VM. Avaliação da satisfação dos usuários em Centros de Atenção Psicossocial. [Internet]. Niterói: Universidade Federal Fluminense: 2018 [cited 2019 Out 04]. Available from: https://app.uff.br/riuff/handle/1/10403

20. Godoy JA, Pavan G, Monteiro RT, Motta LS, Pacheco MA, Nogueira EL, et al. Satisfaction with care in a brazilian psychiatric inpatient unit: differences in perceptions among patients according to type of health insurance. Trends Psychiatry Psychother. [Internet]. 2019 Mar [cited 2019 Oct 03]; 41(1):27-35. Available from: http://www.scielo.br/scielo.php?pid=S2237-60892019000100004Escript=sci_arttext

21. Miller DI. Inpatient Psychiatric Care: Families' Expectations and Perceptions of Support Received From Health Professionals. Perspect Psychiatr Care. [Internet]. 2017. [cited 2019 Set 30]; 53(4):350-6. Available from: https://doi.org/10.1111/ppc.12168

22. Bosque RM, Oliveira MAF de, Silva NN da, Claro HG, Fernandes IF de AL. Satisfação dos Trabalhadores, Usuários e Familiares dos Centros de Atenção Psicossocial em Álcool e Outras Drogas. Rev enferm UFPE [Internet]. 2017 [cited 2018 Mar 10]:11(S11):4598-606. Available from: https://periodicos.ufpe.br/revistas/revistaenfermagem/article/viewFile/231199/25195

23. Santos LA. Satisfação dos familiares com os serviços prestados por um centro de atenção psicossocial infanto-juvenil. [Internet]. São João del-Rei: Universidade Federal de São João del-Rei; 2018 [cited 2019 Set 26]. Available from: https://ufsj.edu.br/portal2-repositorio/File/ ppgpsi/Publicacoes/Dissertacoes/LARISSA\%20ASSUNCAO\%20SANTOS.pdf

24. Soares CGC. Avaliação da satisfação e da sobrecarga de profissionais cuidadores de serviços residenciais terapêuticos. [Internet]. São João del-Rei: Universidade Federal de São João del-Rei; 2016 [cited 2019 Out 02]. Available from: http:// ufsj.edu.br/portal2-repositorio/File/ ppgpsi/Publicacoes/Dissertacoes/CARLOS\%20GUILHERME\%20CRISTELLI\%20SOARES.pdf

25. Costa PHA, Colugnati FAB, Ronzani TM. Avaliação de serviços em saúde mental no Brasil: revisão sistemática da literatura. Cien Saude Colet [Internet]. 2015 Oct [cited 2018 Apr 10];20(10):3243-53. Disponível em: http://www.scielo.br/scielo.php?script=sci_arttextEpi$d=S 1413-81232015001003243 \&$ lng $=p t \& t \operatorname{lng}=p t$ 